\title{
REGULAR PI METRIC FLOWS ARE EQUICONTINUOUS
}

\author{
ELI GLASNER
}

(Communicated by James E. West)

\begin{abstract}
Let $(X, T)$ be a metrizable minimal flow. We show that a homomorphism $X \stackrel{\pi}{\longrightarrow} Y$, which is regular, and PI can be decomposed as $X \stackrel{\sigma}{\longrightarrow}$ $Z \stackrel{\rho}{\longrightarrow} Y, \pi=\rho \circ \sigma$, where $\rho$ is proximal and $\sigma$ is a compact group extension. In particular, assuming further that $T$ is abelian and taking $Y$ to be the trivial one point flow, we find that a metric regular PI flow is a compact group rotation.
\end{abstract}

\section{INTRODUCTION}

By a theorem of W. H. Gottchalk [Go], a metrizable minimal distal regular flow $(X, T)$, where $T$ is abelian, is necessarily a compact group rotation. This was generalized by $\mathrm{J}$. Auslander in $[\mathrm{A}]$ to a general group $T$ and by the author [G1], to point distal regular homomorphisms. Here we prove the following theorem:

Theorem 1. Let $(X, T)$ be a metric minimal flow, $X \stackrel{\pi}{\rightarrow} Y$ a regular PI homomorphism. Then $\pi=\mu \circ \nu$, where $X \stackrel{\nu}{\rightarrow} Z$ is a group homomorphism and $Z \stackrel{\mu}{\longrightarrow} Y$ is proximal. If in addition, $\pi$ is RIC, then $\pi$ is a group homomorphism.

Taking $Y$ as the trivial flow, we get

Corollary 1. If $(X, T)$ is a minimal regular PI metric flow, then $(X, T)$ is a group extension of a proximal flow. If $(X, T)$ is incontractible (as is always the case when $T$ is abelian), then $(X, T)$ is a compact group rotation.

Theorem 4.7 of [G1], which concerns point distal regular open homomorphisms, is a corollary of Theorem 1. (Although in the proof of Theorem 1 we use Proposition 3.1 of [G1], which is about a distal regular homomorphism being a group homomorphism.) However, Theorem 4.5 of [G1], which states that a nontrivial factor of almost simple flow is up to almost 1-1 extension a group homomorphism, cannot be deduced from Theorem 1 because it is not known whether an almost simple flow is almost 1-1 equivalent to a metrizable regular flow.

Examples of regular PI (nonmetrizable) flows that are not equicontinuous are easy to find. One is the enveloping semigroup of the flow on the 2-torus $\mathbb{R}^{2} / \mathbb{Z}^{2}$

Received by the editors June $15,1990$.

1980 Mathematics Subject Classification (1985 Revision). Primary 54H20. 
given by $T(x, y)=(x+\alpha, x+y)[\mathrm{F}, \mathrm{N}]$; another is the "two circle" minimal set $[\mathrm{E}]$.

In $\S 2$ we briefly review the various definitions of the notions mentioned above and describe the algebraic notations and techniques that are used in proving Theorem 1. We also prove some basic lemmas including the crucial Lemma 3 on the topological isomorphism of certain automorphism groups. Surprisingly, the proof of this lemma requires theorems of Souslin and Banach about measurable maps. Section 3 is devoted to the proof of Theorem 2, which asserts the regularity of each of the components of the canonical PI-tower corresponding to a regular homomorphism $X \stackrel{\pi}{\rightarrow} Y$ over the base $Y$.

The last section uses Theorem 2 to reduce the proof of Theorem 1 to the case of a regular distal homomorphism, which by [G1], is necessarily a group homomorphism. Using essentially the same proof, we get Theorem 3 , which says that when $X$ is metric and $X \stackrel{\pi}{\rightarrow} Y$ is RIC and regular, then $\pi=\kappa \circ \omega$, $X \stackrel{\omega}{\rightarrow} Z \stackrel{\kappa}{\rightarrow} Y$, where $Z \stackrel{\kappa}{\rightarrow} Y$ is the largest almost periodic extension (actually a group extension) of $Y$ under $\pi$, and $x \stackrel{\omega}{\rightarrow} Z$ is RIC and weakly mixing.

\section{DEFINITIONS, A REVIEW OF SOME THEOREMS, AND SOME BASIC LEMMAS}

We refer to [E, V, A, G2] for more details on the algebraic theory of minimal flows; we now describe some of its aspects. Assume $T$ to be a discrete group, and as usual, let $\beta T$ denote the Stone-Čech compactification of $T$. Let $M$ be a fixed minimal ideal in $\beta T$ and $J \subset M$ the set of idempotents in $M$. We fix an element $u \in J$, and let $G$ be the group $u M$.

$\beta T$ acts on every flow $(X, T)$ and the orbit closure of a point $x \in X$ is given by $\bar{o}(x)=\{p x: p \in \beta T\}$. A point $x \in X$ is almost periodic (a.p.) if $\bar{o}(x)$ is minimal, iff $\bar{o}(x)=\{p x: p \in M\}$.

A flow $(X, T)$ satisfies the Bronstein condition (B.c.) if the a.p. points are dense in $X$. Given $X \stackrel{\pi}{\rightarrow} Y$, a homomorphism of minimal flows, we let

$$
R_{\pi}=\left\{\left(x, x^{\prime}\right) \in X \times X: \pi(x)=\pi\left(x^{\prime}\right)\right\} .
$$

The homomorphism $\pi$ satisfies $B c$ if $R_{\pi}$ is a $B c$ flow. Let $\Gamma_{\pi}=\{\psi \in$ $\operatorname{Aut}(X, T): \pi \circ \psi=\pi\}$.

Here $\operatorname{Aut}(X, T)$ is the group of all self-homeomorphisms of $X$ such that $\psi \circ t=t \circ \psi \forall t \in T$.

We provide $\operatorname{Aut}(X, T)$ with the topology of uniform convergence of homomorphisms and their inverses. When $X$ is metric, $\operatorname{Aut}(X, T)$ is a polish topological group.

A pointed flow is a flow $(X, T)$ with a distinguished base point $x_{0} \in X$. We adopt the convention of always choosing a base point $x_{0}$ satisfying $u x_{0}=x_{0}$.

When $\left(X, x_{0}\right)$ is a pointed flow its Ellis group is the subgroup $\mathscr{G}(X)=$ $\mathscr{G}\left(X, x_{0}\right)=\left\{\alpha \in G: \alpha x_{0}=x_{0}\right\}$ of $G$.

A homomorphism $\left(X, x_{0}\right) \stackrel{\pi}{\rightarrow}\left(Y, y_{0}\right)$ (this means $\left.\pi x_{0}=y_{0}\right)$ is proximal (i.e. $\pi x=\pi x^{\prime} \Rightarrow x$ and $x^{\prime}$ are proximal) iff $\mathscr{G}\left(X, x_{0}\right)=\mathscr{G}\left(Y, y_{0}\right)$.

For the definition of the $\tau$-topology on $G$, we refer to e.g. [G2]. If $F$ is a $\tau$-closed subgroup of $G$, then

$$
F^{\prime}=\bigcap\{\tau-\operatorname{cl} r(O): O \text { is a } \tau-\operatorname{nbd} \text { of } u \text { in } F\} .
$$

$F^{\prime}$ is a $\tau$-closed subgroup of $F$, which is invariant under all $\tau$-continuous automorphisms of $F$; in particular $F^{\prime} \triangleleft F, F^{\prime \prime} \triangleleft F$ etc. $F / F^{\prime}$ with the quo- 
tient $\tau$-topology is a compact Hausdorff topological group. A homomorphism $\left(X, x_{0}\right) \stackrel{\pi}{\rightarrow}\left(Y, y_{0}\right)$ of minimal flows is almost periodic iff it is distal (i.e. $\pi x=\pi x^{\prime}, x \neq x^{\prime} \Rightarrow x$, and $x^{\prime}$ are not proximal) and $F^{\prime} \subset A$, where $A=\mathscr{G}\left(X, x_{0}\right), F=\mathscr{G}\left(Y, y_{0}\right)$. An almost periodic homomorphism is a group homomorphism if in addition $A \triangleleft F$, and in that case, the topological groups $\Gamma_{\pi}$ and $F / A$ are isomorphic and $(Y, T) \cong\left(X / \Gamma_{\pi}, T\right)$.

A quasifactor of a minimal flow $(X, T)$ is a minimal subflow of the flow $\left(2^{X}, T\right)$ induced on the space $2^{X}$ of closed subsets of $X$ by $T$. For $p \in \beta T$, $K \in 2^{X}$ the action of $p$ on $K$ is written as $p \circ K$. This is to distinguish it from the subset $p K=\{p x: x \in K\}$ of $X$, which is usually not even closed. We have, however, $p K \subset p \circ K$. Given a homomorphism $\left(X, x_{0}\right) \stackrel{\pi}{\rightarrow}\left(Y, y_{0}\right)$ of minimal sets with $\mathscr{G}\left(X, x_{0}\right)=A \subset F=\mathscr{G}\left(Y, y_{0}\right)$, we let $\widetilde{Y}=\left\{p \circ F x_{0}: p \in\right.$ $M\}$. This is a quasifactor of $X$, and we say that $\pi$ is a RIC-homomorphism (or extension) if the sets $p \circ F x_{0}$ are exactly the fibers of the map $\pi$; i.e. $\pi^{-1}\left(p y_{0}\right)=p \circ F x_{0} \forall p \in M$. In any case $\theta: p \circ F x_{0} \mapsto p y_{0} \quad(p \in M)$ is a homomorphism of $\widetilde{Y}$ onto $Y$, and it is an isomorphism iff $\pi$ is RIC. A RIC homomorphism is open and satisfies $B c$. Let $\tilde{y}_{0}=u \circ F x_{0}$ be the base point of $\widetilde{Y}$, and let $\widetilde{X}=X \vee \widetilde{Y}=\bar{o}\left(x_{0}, \tilde{y}_{0}\right) \subset X \times \widetilde{Y}$. Then $\widetilde{X}=\{(x, \tilde{y}): x \in \tilde{y}\}=$ $\left\{\left(p x_{0}, p \circ F x_{0}\right): p \in M\right\}$. If we let $\tilde{\theta}(x, \tilde{y})=\tilde{y}$ and $\tilde{\pi}(x, \tilde{y})=x$, then in the shadow diagram

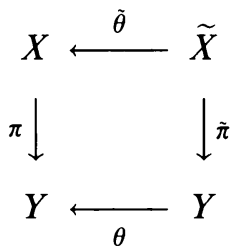

the maps $\theta, \tilde{\theta}$ are proximal and $\tilde{\pi}$ is RIC. When $X \stackrel{\pi}{\rightarrow} Y$ is RIC, a commutative diagram

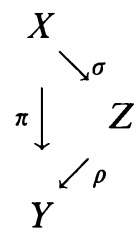

can be constructed such that $\rho$ is almost periodic and $\mathscr{G}\left(Z, z_{0}\right)=F^{\prime} A$.

The latter condition means that $Z$ is the largest almost periodic extension of $Y$ that is a factor of $X$. In particular, when $X$ is metric, $\pi$ is weakly mixing (i.e. $R_{\pi}$ is topologically ergodic) iff $\rho$ is trivial.

The construction of the shadow diagram producing RIC-homomorphisms and the construction of the maximal almost periodic extension within a RIChomomorphism are the basic blocks of the canonical PI-tower associated with a homomorphism $X \stackrel{\pi}{\longrightarrow} Y$. Thus the first stage of this tower is the diagram

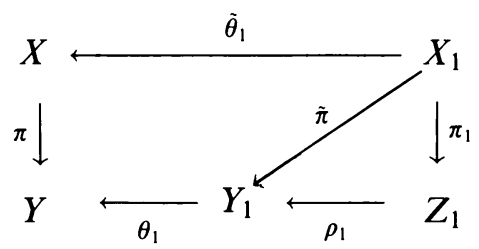


where $Y_{1}=\tilde{Y}, X_{1}=\tilde{X}$, and $Z_{1} \stackrel{\rho_{1}}{\longrightarrow} Y_{1}$ is the largest almost periodic extension of $Y_{1}$ under the RIC extension $\tilde{\pi}$. The next stage is the diagram

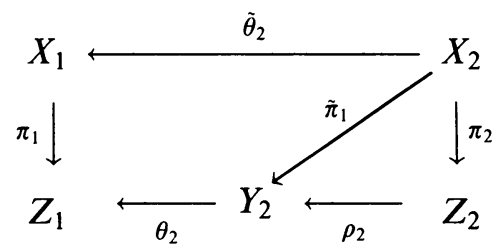

where $\tilde{\pi}_{1}$ is the RIC shadow of $\pi_{1}$ and $\rho_{2}$ the largest almost periodic extension of $Y_{2}$ under $\tilde{\pi}_{1}$ etc.

If necessary, one utilizes inverse limits to construct inductively flows $\left\{Y_{\nu}\right.$, $\left.Z_{\nu}, X_{\nu}: \nu \leq \eta\right\}$, where $\eta$ is the first nonlimit ordinal for which $Z_{\eta}=Y_{\eta}$. The top of the tower consists of the flows $X_{\infty}=X_{\eta}, Y_{\infty}=Y_{\eta}=Z_{\eta}$, where the diagram

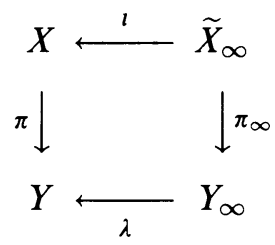

is achieved. Here $\pi_{\infty}$ is RIC and weakly mixing, $Y_{\infty} \stackrel{\lambda}{\rightarrow} Y$ is a strictly PI homomorphism (i.e. can be decomposed into possibly transfinite sequence of alternating proximal and a.p. homomorphism), and $l$ is a proximal extension. When $X$ is metrizable, each flow in the PI-tower is metrizable and the ordinal $\eta$ is countable.

We say that $\pi$ is a PI homomorphism if $\pi_{\infty}$ is an isomorphism. Though we are not going to use it, we mention the following characterization of PI homomorphism due to I. U. Bronstein (see [A]). When $X$ is metric, $X \stackrel{\pi}{\longrightarrow} Y$ is PI iff every closed invariant subset of $R_{\pi}$ that is both topologically ergodic and satisfies $B C$ is minimal.

A homomorphism $X \stackrel{\pi}{\longrightarrow} Y$ of minimal flows is called regular if whenever $\left(x, x^{\prime}\right) \in R_{\pi}$ is an a.p. point, there exists an automorphism $\psi \in \Gamma_{\pi}$ with $x^{\prime}=$ $\psi(x)$. $X$ is called regular if this condition holds for every a.p. $\left(x, x^{\prime}\right) \in X \times X$.

Lemma 1. Let $\left(X, x_{0}\right) \stackrel{\pi}{\rightarrow}\left(Y, y_{0}\right)$ be a regular homomorphism of minimal flows. Let $A=\mathscr{G}\left(X, x_{0}\right), F=\mathscr{G}\left(Y, y_{0}\right)$, then $A \triangleleft F$ and there exists $a$ natural algebraic homomorphism $\gamma \rightarrow \psi_{\gamma}$ of $F$ onto $\Gamma_{\pi}$ with kernel $A$. Thus $\Gamma_{\pi}$ is algebraically isomorphic to $F / A$.

Proof. Let $\gamma \in F$; then $\left(x_{0}, \gamma x_{0}\right)$ is an almost periodic point in $R_{\pi}$. By the regularity of $\pi, \exists \psi_{\gamma} \in \Gamma_{\pi}$ for which $\psi_{\gamma}^{-1}\left(x_{0}\right)=\gamma x_{0}$. For $p \in M$ we have

$$
\psi_{\gamma}^{-1}\left(p x_{0}\right)=p \psi^{-1}\left(x_{0}\right)=p \gamma x_{0} .
$$

If $\delta \in F$, then $\psi_{\gamma \delta}^{-1}\left(p x_{0}\right)=p \gamma \delta x_{0}=\psi_{\delta}^{-1}\left(p \gamma x_{0}\right)=\psi_{\delta}^{-1} \circ \psi_{\gamma}^{-1}\left(p x_{0}\right)=$ $\left(\psi_{\gamma} \circ \psi_{\delta}\right)^{-1}\left(p x_{0}\right)$ and $\gamma \mapsto \psi_{\gamma}$ is a homomorphism. Clearly the kernel of this homomorphism is $A$. Finally if $\psi \in \Gamma_{\pi}$ then $u \psi\left(x_{0}\right)=\psi\left(u x_{0}\right)=\psi\left(x_{0}\right)$, and therefore, $\psi\left(x_{0}\right)=\gamma^{-1} x_{0}$ for some $\gamma \in G$. Since $\gamma^{-1} y_{0}=\gamma^{-1} \pi\left(x_{0}\right)=$ $\pi\left(\gamma^{-1} x_{0}\right)=\pi \circ \psi\left(x_{0}\right)=\pi\left(x_{0}\right)=y_{0}$, we have $\gamma \in F$ and $\psi=\psi_{\gamma}$. 
Lemma 2. In the diagram

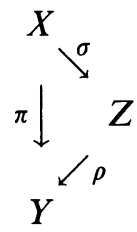

let $\rho$ be proximal. Then $\Gamma_{\pi}=\Gamma_{\sigma}$.

Proof. Suppose $\psi \in \Gamma_{\sigma}$ i.e. $\sigma \circ \psi=\sigma$. Then $\pi \circ \psi=\rho \circ \sigma \circ \psi=\rho \circ \sigma=\pi$ and $\psi \in \Gamma_{\pi}$. Thus $\Gamma_{\sigma} \subset \Gamma_{\pi}$. On the other hand, if $\psi \in \Gamma_{\pi}$, then for $x \in X$, $\rho(\sigma(\psi(x)))=\pi \circ \psi(x)=\pi x=\rho(\sigma x)$, and we conclude that $\sigma(\psi(x))$ and $\sigma(x)$ are proximal. However, $(x, \psi(x))$ is an a.p. point and therefore, so is $(\sigma x, \sigma(\psi(x)))$. This can happen only when $\sigma x=\sigma(\psi x)$, and since $x$ is an arbitrary point of $X$, we have $\sigma=\sigma \circ \psi$, so that $\psi \in \Gamma_{\sigma}$ and $\Gamma_{\pi} \subset \Gamma_{\sigma}$.

Lemma 3. In the diagram

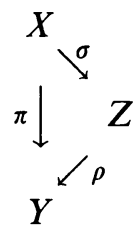

suppose $\pi$ and $\rho$ are regular, $\sigma$ is proximal, and $X$ is metrizable. Then $\Gamma_{\pi}$ and $\Gamma_{\rho}$ are topologically isomorphic.

Proof. Since $\sigma$ is proximal $\mathscr{G}\left(X, x_{0}\right)=\mathscr{G}\left(Z, z_{0}\right)=A$. Thus by Lemma $1, \Gamma_{\pi}, \Gamma_{\rho}$, and $F / A$ where $F=\mathscr{G}\left(Y, y_{0}\right)$, are all algebraically isomorphic. Moreover, it is clear that the canonical isomorphism $J: \Gamma_{\pi} \rightarrow \Gamma_{\rho}$ is continuous. Since $\Gamma_{\pi}$ and $\Gamma_{\rho}$ are polish groups and $J$ is $1-1$, by a theorem of Souslin [K, Vol. I, p. 487], $J$ is a Borel isomorphism. In particular, $J^{-1}: \Gamma_{\rho} \rightarrow \Gamma_{\pi}$ is a Borel measurable homomorphism of polish groups. By a theorem of Banach [B], such a homomorphism is continuous, and we conclude that $J$ is a topological isomorphism as claimed.

\section{THE PI-TOWER FOR A REGULAR HOMOMORPHISM}

Theorem 2. Let $X \stackrel{\pi}{\longrightarrow} Y$ be a regular homomorphism of minimal flows; then each of the homomorphisms onto $Y$ of the flows $X_{\nu}, Y_{\nu}, Z_{\nu}(\nu \leq \eta)$ constructed in the canonical PI-tower for $\pi$ is regular.

The proof is a consequence of the following three lemmas.

Lemma 4. Let

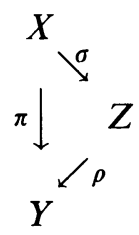


be a diagram where $\pi$ and $\rho$ are regular, and let

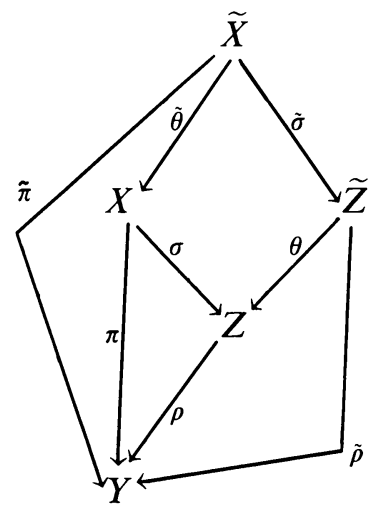

be the same diagram with the attached RIC shadow diagram for $\pi$. Then the maps $\tilde{\pi}$ and $\tilde{\rho}$ are regular.

Proof. Denote $A=\mathscr{G}\left(X, x_{0}\right), F=\mathscr{G}\left(Y, y_{0}\right)$, and $B=\mathscr{G}\left(Z, z_{0}\right)$, then Lemma 1 implies $A \triangleleft F$ and $B \triangleleft F$. We recall the definitions $\widetilde{Z}=\left\{p \circ B x_{0}: p \in\right.$ $M\}, \tilde{z}_{0}=u \circ B x_{0}$, and $\widetilde{X}=\left\{p\left(x_{0}, u \circ B x_{0}\right): p \in M\right\}=\{(x, \tilde{z}): x \in \tilde{z} \in \widetilde{Z}\}$. Given $\psi \in \Gamma_{\pi}$, we define a map $\Psi: \widetilde{Z} \rightarrow 2^{X}$ by $\Psi\left(p \circ B x_{0}\right):=\psi\left(p \circ B x_{0}\right)=$ $p \circ B \psi x_{0}$. If $\psi\left(x_{0}\right)=\gamma x_{0}$ for $\gamma \in G$, then

$$
\gamma y_{0}=\gamma \pi\left(x_{0}\right)=\pi\left(\gamma x_{0}\right)=\pi \circ \psi\left(x_{0}\right)=\pi\left(x_{0}\right)=y_{0}
$$

so that $\gamma \in F$. Now $\Psi\left(p \circ B x_{0}\right)=p \circ B \psi\left(x_{0}\right)=p \circ \gamma B x_{0}=p \gamma \circ B x_{0} \in \tilde{Z}$, and $\Psi$ maps $\tilde{Z}$ into itself. Moreover, if also $\psi\left(x_{0}\right)=\delta x_{0}$ for $\delta \in F$, then $\gamma x_{0}=\delta x_{0}$ implies $\gamma^{-1} \delta \in A \subset B$ and $\delta B=\gamma B$. Thus for every $p \in M$,

$$
p \gamma \circ B x_{0}=p \circ \gamma B x_{0}=p \circ \delta B x_{0}=p \delta \circ B x_{0},
$$

and $\Psi$ is well defined. It is also clear now that $\Psi$ is a continuous automorphism of $(\tilde{Z}, T)$ where the inverse $\Psi^{-1}$ is induced by $\psi^{-1}$. We now define a map $\tilde{\psi}: X \times \widetilde{Z} \rightarrow X \times \widetilde{Z}$ by $\tilde{\psi}(x, \tilde{z})=(\psi(x), \Psi(\tilde{z}))$. Since for $p \in M$

$$
\tilde{\psi}\left(p\left(x_{0}, \tilde{z}_{0}\right)\right)=p\left(\psi\left(x_{0}\right), \Psi\left(u \circ B x_{0}\right)\right)=p\left(\gamma x_{0}, \gamma \circ B x_{0}\right)=p \gamma\left(x_{0} \tilde{z}_{0}\right) \in \tilde{X},
$$

we see that $\tilde{\psi}$ is an automorphism of $(\tilde{X}, T)$.

Now let $\left((x, \tilde{z}),\left(x^{\prime}, \tilde{z}^{\prime}\right)\right)$ be an a.p. point of $R_{\tilde{\pi}}$. Then there exist an idempotent $v \in J$ and $\delta, \zeta \in G$ such that

$$
(x, \tilde{z})=v \zeta\left(x_{0}, \tilde{z}_{0}\right), \quad\left(x^{\prime}, \tilde{z}^{\prime}\right)=v \delta\left(x_{0}, \tilde{z}_{0}\right) .
$$

Clearly $\zeta y_{0}=\delta y_{0}$ and therefore, $\zeta^{-1} \delta \in F$. Now $\left(x, x^{\prime}\right)$ is an a.p. point of $R_{\pi}$ and by regularity of $\pi$, a $\psi \in \Gamma_{\pi}$ exists for which $x^{\prime}=\psi(x)$. Let $\xi \in F$ be such that $\psi\left(x_{0}\right)=\xi x_{0}$; then $\Psi\left(v \zeta \circ B x_{0}\right)=v \zeta \xi \circ B x_{0}$ and $v \delta x_{0}=$ $x^{\prime}=\psi(x)=\psi\left(v \zeta x_{0}\right)=v \zeta \psi\left(x_{0}\right)=v \zeta \xi x_{0}$, implies $\delta^{-1} \zeta \xi \in A \subset B$. Thus $\delta^{-1} \zeta \xi \circ B x_{0}=u \circ B x_{0}$, hence $\zeta \xi \circ B x_{0}=\delta \circ B x_{0}$ and $\Psi(\tilde{z})=\psi\left(v \zeta \circ B x_{0}\right)=$ $v \zeta \xi \circ B x_{0}=v \delta \circ B x_{0}=\tilde{z}^{\prime}$. It follows that $\tilde{\psi}(x, \tilde{z})=\left(x^{\prime}, \tilde{z}^{\prime}\right)$ and $\tilde{\pi}$ is regular.

Finally if $\left(\tilde{z}, \tilde{z}^{\prime}\right)=\left(v \zeta \tilde{z}_{0}, v \delta \tilde{z}_{0}\right)$ is an a.p. point in $R_{\tilde{\rho}}$, then $((x, \tilde{z})$, $\left.\left(x^{\prime}, \tilde{z}^{\prime}\right)\right)=\left(\left(v \zeta x_{0}, v \zeta \tilde{z}_{0}\right),\left(v \delta x_{0}, v \delta \tilde{z}_{0}\right)\right)$ is an a.p. point in $R_{\tilde{\pi}}$ and $\tilde{z}^{\prime}=\Psi(\tilde{z})$ where $\Psi \in \Gamma_{\tilde{\rho}}$ is constructed as above. This proves that also $\tilde{\rho}$ is regular. 
Lemma 5. In the diagram

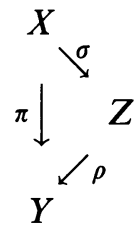

suppose $\rho$ is regular and $\sigma$ is distal. If $\mathscr{G}\left(X, x_{0}\right)=A \triangleleft F=\mathscr{G}\left(Y, y_{0}\right)$, then $\pi$ is regular

Proof. Given $\gamma \in F$, by Lemma 1 there exists an automorphism $\psi_{\gamma} \in \Gamma_{\rho}$ defined by $\psi_{\gamma}\left(p z_{0}\right)=p \gamma z_{0} \quad(p \in M)$. Define $\psi_{\gamma} \in \Gamma_{\pi}$ by $\psi_{\gamma}\left(p x_{0}\right)=p \gamma z_{0}$. We first show that $\psi_{\gamma}$ is well defined on $X$. Suppose $p x_{0}=q x_{0}, p, q \in M$. Then a fortiori $p z_{0}=q z_{0}$ and $\psi_{\gamma}\left(p z_{0}\right)=p \gamma z_{0}, \psi_{\gamma}\left(q z_{0}\right)=q \gamma z_{0}$ implies $p \gamma z_{0}=q \gamma z_{0}$. Hence

$$
\sigma\left(q \gamma x_{0}\right)=q \gamma \sigma\left(x_{0}\right)=q \gamma z_{0}=p \gamma z_{0}=p \gamma \sigma\left(x_{0}\right)=\sigma\left(p \gamma x_{0}\right) .
$$

If $v \in J$ satisfies $v p=p$, then

$$
v \sigma\left(p \gamma x_{0}\right)=v p \gamma z_{0}=p \gamma z_{0}=\sigma\left(p \gamma x_{0}\right),
$$

and the distality of $\sigma$ implies $v q \gamma x_{0}=q \gamma x_{0}$ as well.

Denote $\alpha=(u p)^{-1}(u q)$, then $\alpha \in A$. Since $A \triangleleft F, \gamma A=A \gamma$, and there exists $\alpha^{\prime} \in A$ with $\gamma \alpha^{\prime}=\alpha \gamma$. Thus $p \gamma x_{0}=v p \gamma \alpha^{\prime} x_{0}=v(u p) \alpha \gamma x_{0}=v(u q) \gamma x_{0}=$ $q \gamma x_{0}$ and $\psi_{\gamma}$ is well defined on $X$. It is clearly an element of $\Gamma_{\pi}$, and if $\left(v \zeta x_{0}, v \xi x_{0}\right)$ is an a.p. point of $R_{\pi}$, then $\gamma=\zeta^{-1} \xi \in F$ and $\psi_{\gamma}\left(v \zeta x_{0}\right)=$ $v \zeta \gamma x_{0}=v \xi x_{0}$ so that $\pi$ is regular.

Lemma 6. If $\left(X_{\nu}, x_{\nu}\right) \stackrel{\pi_{\nu}}{\longrightarrow}\left(Y, y_{0}\right)$ is an inverse system of regular homomorphisms of pointed minimal flows, then $\lim _{\longleftarrow} X_{\nu}=X \stackrel{\pi}{\rightarrow} Y$ is also regular.

Proof. If $\gamma \in F=\mathscr{G}\left(Y, y_{0}\right)$, then by Lemma $1 \psi_{\gamma}\left(p x_{\nu}\right)=p \gamma x_{\nu} \quad(p \in M)$ defines an automorphism on each $X_{\nu}$. This and the definition of the inverse limit automatically define $\psi_{\gamma}$ on $X$ as well. If $\left(x, x^{\prime}\right) \in R_{\pi}$ is an almost periodic point, then for some $v \in J, \zeta, \xi \in F, x=v \zeta x_{0}, x^{\prime}=v \xi x_{0}$ where $x_{0} \in X$ is the point corresponding to the points $x_{\nu} \in X_{\nu}$. If we let $\gamma=\zeta^{-1} \xi \in F$, then clearly $\psi_{\gamma}(x)=x^{\prime}$.

The proof of Theorem 2 follows by transfinite induction on the canonical PI-tower for $X \stackrel{\pi}{\longrightarrow} Y$, using Lemmas 4, 5, and 6. For the use of Lemma 5, we recall that $F^{\nu} \triangleleft F$ for all $\nu$, where $F^{\nu+1}=\left(F^{\nu}\right)^{\prime}$, and that therefore, also $\mathscr{G}\left(Z_{\nu}, z_{\nu}\right)=F^{\nu} A \triangleleft F$.

\section{A PROOF OF THEOREM 1}

We are given a metric minimal flow $X$ and a regular PI homomorphism $X \stackrel{\pi}{\longrightarrow} Y$. Thus in the canonical PI-tower for $\pi, X_{\infty}=Y_{\infty}$. In the notations of $\S 2$, consider the chain

$$
Z_{2} \stackrel{\rho_{2}}{\longrightarrow} Y_{2} \stackrel{\theta_{2}}{\longrightarrow} Z_{1} \stackrel{\rho_{1}}{\longrightarrow} Y_{1} \stackrel{\theta_{1}}{\longrightarrow} Y .
$$

We have $\mathscr{G}(Y)=\mathscr{G}\left(Y_{1}\right)=F, \mathscr{G}\left(Z_{1}\right)=\mathscr{G}\left(Y_{2}\right)=F^{\prime} A$, and $\mathscr{G}\left(Z_{2}\right)=F^{\prime \prime} A$, where by Lemma $1, \mathscr{G}(X)=A \triangleleft F$. 
Let $\theta=\theta_{1} \circ \rho_{1} \circ \theta_{2}$ and $\rho=\theta_{1} \circ \rho_{1}$. By Theorem 2, $\rho$ and $\theta$ are regular, and by Lemma 3, $\Gamma_{\rho}$ and $\Gamma_{\theta}$ are topologically isomorphic. By Lemma $2, \Gamma_{\rho}=\Gamma_{\rho_{1}}$. Now since $A \triangleleft F, F^{\prime} A \triangleleft F$, the homomorphism $\rho_{1}$ is a group homomorphism. Thus the topological group $\Gamma_{\rho_{1}}$ is a compact group (topologically isomorphic to the compact Hausdorff topological group $F / F^{\prime} A$ with its $\tau$-topology). We also conclude that $\Gamma_{\theta}$ is a compact group. Let $W$ be the quotient flow $Y_{2} / \Gamma_{\theta}$ and let

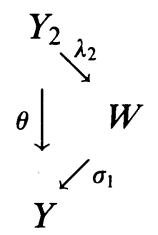

be the associated diagram. $\mathscr{G}(W) \subset F$ and since every $\gamma \in F$ defines an element $\psi_{\gamma} \in \Gamma_{\theta}$, we also have $F \subset \mathscr{G}(W)$. Thus $\mathscr{G}(W)=F=\mathscr{G}(Y)$ and $\lambda_{1}$ is proximal. We now have the alternative chain

$$
Z_{2} \stackrel{\rho_{2}}{\longrightarrow} Y_{2} \stackrel{\lambda_{2}}{\longrightarrow} W \stackrel{\lambda_{1}}{\longrightarrow} Y,
$$

where $\rho_{2}$ and $\lambda_{2}$ are group extensions and $\lambda_{1}$ is proximal. Put $\kappa_{1}=\lambda_{1} \circ \lambda_{2} \circ \rho_{2}$ and $\kappa_{2}=\lambda_{2} \circ \rho_{2}$. Then $\kappa_{2}$ is a distal homomorphism.

If $\left(x, x^{\prime}\right)$ is an a.p. point in $R_{\kappa_{2}} \subset R_{\kappa_{1}}$ then by regularity of $\kappa_{1}$ (Theorem 2) $\exists \psi \in \Gamma_{\kappa_{1}}$ with $x^{\prime}=\psi(x)$. However, by Lemma 2, $\Gamma_{\kappa_{2}}=\Gamma_{\kappa_{1}}$ so that $\psi \in \Gamma_{\kappa_{2}}$ and we conclude that $\kappa_{2}$ is also regular. It follows from [G1, Proposition 3.1] that $Z_{2} \stackrel{\kappa_{2}}{\longrightarrow} W$ is almost periodic, and since $\mathscr{G}\left(Z_{2}\right)=F^{\prime \prime} A, \mathscr{G}(W)=F$, this implies $F^{\prime} \subset F^{\prime \prime} A$. Since $\mathscr{G}\left(Y_{2}\right)=F^{\prime} A$, we now have $\mathscr{G}\left(Z_{2}\right)=F^{\prime \prime} A=F^{\prime} A=$ $\mathscr{G}\left(Y_{2}\right)$, whence $Y_{2}=Z_{2}=Y_{\infty}=X_{\infty}$. Thus our PI-tower is of height two and we have the following diagram

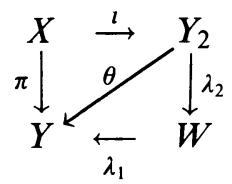

where $l$ is proximal. Apply Lemma 3 again to the diagram<smiles></smiles>

to get the topological isomorphism of the compact $\Gamma_{\theta}$ with $\Gamma_{\pi}$. Thus $\Gamma_{\pi}$ is also compact and denoting the quotient flow $X / \Gamma_{\pi}$ by $L$ and the quotient map $X \rightarrow L$ by $\nu$. We find that the map $L \stackrel{\mu}{\rightarrow} Y$ is proximal.

Thus $\pi=\mu \circ \nu$ is the required decomposition. It is easy to check that when $\pi$ is RIC $\mu$ must be trivial and $\pi=\nu$ is a group homomorphism. This completes the proof of Theorem 1 .

Theorem 3. Let $(X, T)$ be a metrizable minimal flow and $X \stackrel{\pi}{\rightarrow} Y$ a regular RIC homomorphism. Let $X \stackrel{\omega}{\rightarrow} Z \stackrel{\kappa}{\rightarrow} Y, \pi=\kappa \circ \omega$, where $Z$ is the largest equicontinuous extension of $Y$ under $\pi$. Then $\omega$ is RIC and weakly mixing, and $\kappa$ is a group homomorphism. In particular, when $X$ is regular and $T$ 
abelian, the homomorphism $X \stackrel{\omega}{\longrightarrow} Z$ of $X$ onto its largest equicontinuous factor is RIC and weakly mixing and $Z$ is a compact group rotation.

Proof. Repeating the proof of Theorem 1 without the assumption $X_{\infty}=Y_{\infty}$, we arrive at the diagram

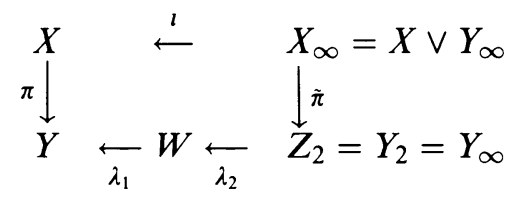

where $\lambda_{1}$ is proximal, $\lambda_{2}$ a group homomorphism, and $\tilde{\pi}$ RIC and weakly mixing. From the simple structure of $Y_{\infty}$ over $Y$ we immediately deduce (in the notation of the canonical tower of $\S 2$ ) that $W=Y_{1}=\tilde{Y}$ and $Z_{2}=Z_{1}$. However, the assumption that $\pi$ is RIC means that $\tilde{Y}=Y=W$, and therefore, $Z=Z_{2}$ is the largest almost periodic extension of $Y$ that is a factor of $X$. Hence $X_{\infty}=X \vee Y_{\infty}=X \vee Z=X$. Put $\omega=\tilde{\pi}$ and $\kappa=\lambda_{2}$ to get the decomposition $\pi=\kappa \circ \omega$.

\section{REFERENCES}

[A] J. Auslander, Minimal flows and their extensions, North-Holland, Amsterdam, 1988.

[B] S. Banach, Théorie des opérations linéaires, Chelsea, New York, 1963.

[E] R. Ellis, Lectures on topological dynamics, Benjamin, New York, 1969.

[F] H. Furstenberg, The structure of distal flows, Amer. J. Math. 85 (1963), 477-515.

[G1] S. Glasner, A topological version of a theorem of Veech and almost simple flows, Ergodic Theory Dynamical Systems 10, (1990), 463-482.

[G2] _ Proximal flows, Lecture Notes in Math., vol. 517, Springer-Verlag, New York, 1976.

[Go] W. H. Gottschalk, Transitivity and equicontinuity, Bull. Amer. Math. Soc. 54 (1948), $982-$ 984.

[K] K. Kuratowski, Topology, vol. I., Warsaw 1948.

[N] I. Namioka, Ellis groups and compact right topological groups, Conference in Modern Analysis and Probability, Contemp. Math., vol. 26, Amer. Math. Soc., Providence, RI, 1984, pp. 295-300.

[V] W. A. Veech, Topological dynamics, Bull. Amer. Math. Soc. 83 (1977), 775-830.

School of Mathematical Sciences, The Raymond and Beverly Sackler faculty of Exact Sciences, Tel Aviv University, Tel Aviv, Israel 